\title{
Exponential Metric in Binary Mass Systems Gravitational Field
}

\author{
N. Perković, M. Stojić
}

\begin{abstract}
This paper deals with the procedure for determining binary mass systems metric. It is performed by placing a test mass $m$ in the common gravitational field of two other objects having much larger mass. The resulting gijmetric tensor is diagonal with the exponential component. Metric tensor exponential components show that the metric in binary systems gravitational field equals the product of metrics of objects making up the system.
\end{abstract}

Index Terms - exponential metrics, mass systems.

\section{INTRODUCTION}

A relativistic approach in the study of the gravitational field of double stars, or binary systems of heavy masses, leads to exponential metric as an apparent metric of Newtonian gravity.

This theoretical concept is based on the application of Newton's law of gravity using the Einstein mass-energy equivalence, i.e. $\mathrm{E}=\mathrm{mc}^{2}[1,2,3]$.

This is achievedby considering the relativistic motion of a body of point mass $\mathbf{m}$ in the gravitational field of the binary system of masses $\mathrm{M}_{1}$ and $\mathrm{M}_{2}$, where $\mathrm{m} \ll \mathrm{M}_{1} \mathrm{i} \mathrm{m} \ll \mathrm{M}_{2}$.

The position of an arbitrary point $\mathbf{P}$ in which the body of mass $\mathbf{m}$ location is determined by the radius vector in the Cartesian coordinate system, which is moved to the center of mass $M_{1}$ and $\mathrm{M}_{2}$ (Figure 1),

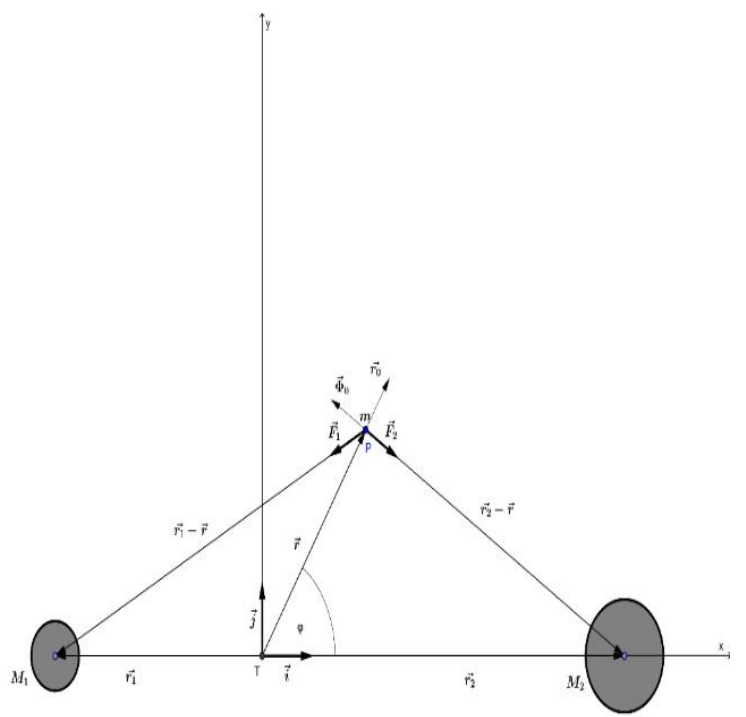

Figure 1
The gravitational field of mass $\mathrm{M}_{1}$ at point Pis

$$
\overrightarrow{g_{1}}=\frac{R_{1} c^{2}}{\left|\vec{r}_{1}-\vec{r}\right|^{3}}\left(\vec{r}_{1}-\vec{r}\right)
$$

while the gravitational field of mass $\mathrm{M}_{2}$ :

$$
\overrightarrow{g_{2}}=\frac{R_{2} c^{2}}{\left|\vec{r}_{2}-\vec{r}\right|^{3}}\left(\vec{r}_{2}-\vec{r}\right)
$$

The gravitational radii $R_{1}$ and $R_{2}$ are defined by the expressions:

$$
\begin{gathered}
R_{1}=\frac{G M_{1}}{c^{2}}(3 a) \\
R_{2}=\frac{G M_{2}}{c^{2}}(3 \mathrm{~b})
\end{gathered}
$$

The total gravitational field at point $\mathbf{P}$ will be the vector sum of the gravitational fields, $\overrightarrow{g_{1}} i \overrightarrow{g_{2}}$

i.e.

$$
\overrightarrow{g_{R}}=\overrightarrow{g_{1}}+\overrightarrow{g_{2}}
$$

The resultant gravitational force acting on mass $\mathbf{m}$ at point $\mathbf{P}$ is

$$
\vec{F}_{R}=m \overrightarrow{g_{R}}=m c^{2}\left[\frac{R_{1}\left(\vec{r}_{1}-\vec{r}\right)}{\left|\vec{r}_{1}-\vec{r}\right|^{3}}+\frac{R_{2}\left(\vec{r}_{2}-\vec{r}\right)}{\left|\vec{r}_{2}-\vec{r}\right|^{3}}\right]
$$

\section{BINARY MASS SYSTEM ENERGY DETERMINATION}

The potential energy change of $\mathrm{M}_{1}$ and $\mathrm{M}_{2}$ coupled system equalsthe work of force $\vec{F}_{R}$ in the gravitational field $\vec{g}_{R}$, i.e.

$$
d U=-\vec{F}_{R} \cdot d \vec{r}=-\vec{F}_{R}\left(\vec{r}_{0} d \vec{r}+\mathrm{r} \vec{\phi}_{0} d \varphi\right)(6)
$$


Using relation (5), equation (6) is obtained:

$$
d U=-m c^{2}\left[R_{1} \frac{-r_{1} \cos (\varphi) d r+r_{1} r \sin (\varphi) d \varphi-r d r}{r_{1}{ }^{3}\left(1-\frac{2 r}{r_{1}} \cos (\varphi)+\frac{r^{2}}{r_{1}^{2}}\right)^{3 / 2}}+R_{2} \frac{r_{2} \cos (\varphi) d r-r_{2} r \sin (\varphi) d \varphi-r d r}{r_{2}{ }^{3}\left(1+\frac{2 r}{r_{2}} \cos (\varphi)+\frac{r^{2}}{r_{2}{ }^{2}}\right)^{3 / 2}}\right] \text { (7) }
$$

Since

$$
c^{2} d m=-d U=-\frac{\partial U}{\partial r} d r-\frac{\partial U}{\partial \varphi} d \varphi(8)
$$

it follows that

is

$$
d(\ln m)=P(r, \varphi) d r+Q(r, \varphi) d \varphi(9)
$$

$$
\begin{aligned}
& P(r, \varphi)=-\frac{R_{1} r_{1}^{2}\left(\frac{\cos (\varphi)}{r_{1}}+\frac{r}{r_{1}^{2}}\right)}{r_{1}^{3}\left(1+\frac{2 r}{r_{1}} \cos (\varphi)+\frac{r^{2}}{r_{1}^{2}}\right)^{3 / 2}}-\frac{R_{2} r_{2}^{2}\left(-\frac{\cos (\varphi)}{r_{2}}+\frac{r}{r_{2}^{2}}\right)}{r_{1}^{3}\left(1-\frac{2 r}{r_{2}} \cos (\varphi)+\frac{r^{2}}{r_{2}^{2}}\right)^{3 / 2}}(10 a) \\
& Q(r, \varphi)=\frac{R_{1} r_{1} \operatorname{rsin}(\varphi)}{r_{1}^{3}\left(1-\frac{2 r}{r_{1}} \cos (\varphi)+\frac{r^{2}}{r_{1}^{2}}\right)^{3 / 2}}-\frac{R_{2} r_{2} \operatorname{rsin}(\varphi)}{r_{1}^{3}\left(1+\frac{2 r}{r_{2}} \cos (\varphi)+\frac{r^{2}}{r_{2}^{2}}\right)^{3 / 2}}(10 b)
\end{aligned}
$$

Integrationof equation (9) gives

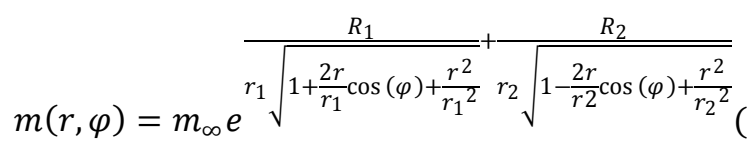

Where $m(r, \varphi)=m_{\infty}$, when $\vec{r} \rightarrow \infty[4,5]$.

By introducing the following function

equation (11) is written in the form

$$
f(r, \varphi)=\frac{R_{1}}{r_{1} \sqrt{1+\frac{r^{2}}{r_{1}^{2}}-\frac{2 r}{r_{1}}} \cos (\varphi)}+\frac{R_{2}}{r_{2} \sqrt{1+\frac{r^{2}}{r_{2}^{2}}+\frac{2 r}{r_{2}}} \cos (\varphi)}
$$

$$
m=m_{\infty} e^{f(r, \varphi)}(13)
$$

because

The total differential of the function is

$$
\begin{aligned}
& \frac{\partial f(r, \varphi)}{\partial r}=P(r, \varphi)(14 a) \\
& \frac{\partial f(r, \varphi)}{\partial \varphi}=Q(r, \varphi)(14 b)
\end{aligned}
$$

$$
d f(r, \varphi)=P(r, \varphi) d r+Q(r, \varphi) d \varphi(15)
$$

The change in gravitational potential of energy systems is defined using equations (8) and (13), giving

$$
d U=-d\left[m_{0} c^{2} e^{f(r, \varphi)}\right](16)
$$

By integrating the equations using the realconditions $U(\infty)=0$, when $\vec{r} \rightarrow \infty$, the gravitational potential energy of the binary mass system is obtained:

$$
U(r, \varphi)=-m c^{2}\left[1-e^{-f(r, \varphi)}\right](17)
$$

The total energy differential of the system is equal to the sum of the differentials of kinetic and gravitational potential energy:

$$
\left.d E=d E_{k}+d U=-d\left[m c^{2}-m c^{2}\left(1-e^{-f(r, \varphi)}\right)\right]=d\left[m c^{2} e^{-f(r, \varphi)}\right)\right](18)
$$

By grouping equations (18), the total energy of the binary system is obtained:

$$
E=m c^{2} e^{-f(r, \varphi)}(19)
$$

Applying the relativistic relations between energy and momentum, the total energy (19) through the Hamiltonian is as follows 
From the Hamiltonian canon equation

$$
\begin{gathered}
H(\vec{r}, \vec{p})=e^{-f(r, \varphi)} \sqrt{g_{\alpha \beta} p^{\alpha} p^{\beta} c^{2}+\mathrm{m}_{0}^{2} c^{4}}(20) \\
\alpha, \beta=1,2,3
\end{gathered}
$$

$$
\frac{\partial H(\vec{r}, \vec{p})}{\partial p^{\alpha}}=\dot{q}_{\alpha}=\frac{p_{\alpha}}{m} e^{-f(r, \varphi)}(21)
$$

the momentum $\mathrm{p}_{\alpha}$, is obtained, i.e.

$$
p_{\alpha}=m \dot{q}_{\alpha} e^{f(r, \varphi)}(22)
$$

It is possible to examine the description of a binary system using a Lagrange formalism whose base consists of generalized coordinates:

and by applying the Lagrange transform:

$$
\begin{gathered}
L\left(q^{i}, \dot{q}^{i}\right)=-m_{0} c \frac{d s}{d t}=-m_{0} c \sqrt{-g_{i j} \frac{d q^{i}}{d t} \frac{d q^{j}}{d t}}(23) \\
i, j=0,1,2,3
\end{gathered}
$$

$$
\begin{gathered}
p_{\alpha} \dot{q}_{\alpha}-H\left(q^{\alpha}, p^{\alpha}\right)=L\left(q^{\alpha}, \dot{q}_{\alpha}\right)(24) \\
\alpha=1,2,3
\end{gathered}
$$

a line element is obtained

$$
d s^{2}=c^{2} d t^{2} e^{-2 f(r, \varphi)}-q_{\alpha \beta} d q^{\alpha} d q^{\beta}(25)
$$

The metric tensor $\mathbf{g}_{\mathrm{ij}}$ is the diagonal whose components are

$$
\begin{array}{ll}
g_{0 \alpha}=0 & \\
g_{11} & =e^{2 f(r, \varphi)} \\
g_{22} & =r^{2} e^{2 f(r, \varphi)}
\end{array} \quad g_{00}=-e^{-2 f(r, \varphi)}(26)
$$

The Yilmaz metric has a linear element

$$
d s^{2}=e^{\Phi} c^{2} d t^{2}-e^{-\Phi}\left(d x^{2}+d y^{2}+d z^{2}\right)
$$

If the function $\mathrm{f}(\mathrm{r}, \varphi)$ according to equation (12) is written in the form:

then

$$
f(r, \varphi)=f_{1}(r, \varphi)+f_{2}(r, \varphi)(28)
$$

$$
\begin{aligned}
& f_{1}(r, \varphi) \frac{R_{1}}{r_{1} \sqrt{1-\frac{2 r}{r_{1}} \cos (\varphi)+\frac{r^{2}}{r_{1}^{2}}}}(29 a) \\
& f_{2}(r, \varphi)=\frac{R_{2}}{r_{2} \sqrt{1+\frac{2 r}{r_{2}} \cos (\varphi)+\frac{r^{2}}{r_{2}^{2}}}}
\end{aligned}
$$

then the components of the $\mathrm{g}_{\mathrm{ij}}$ metric tensor can be written in the form:

$$
\begin{aligned}
& g_{0 \alpha}=0 \\
& g_{00}=-e^{-2 f_{1}(r, \varphi)} e^{-2 f_{2}(r, \varphi)} \quad g_{11}=e^{2 f_{1}(r, \varphi)} e^{2 f_{2}(r, \varphi)}(30) \\
& g_{22}=r^{2} e^{2 f_{1}(r, \varphi)} e^{2 f_{2}(r, \varphi)}
\end{aligned}
$$

From equations (28), it can be concluded that the metric of linear mass systems is equal to the product of metrics of the system components.

\section{CONCLUSION}

A relativistic approach in the study of the gravitational field of binary meat systems leads to an exponential metric. Metric results obtained, $\mathrm{g}_{\mathrm{ij}}$ is diagonal.Diagonal metric tensor components show that the metric in the gravitational field of binary systems is equal to the product of the metric of the system components.

\section{REFERENCES}

[1] L. D. Landau and E. M. Lifshitz: The Classical Theory of Fields, Pergamon Press, 1971

[2] A. Einstein: The Meaning of Relativity, Princeton University Press, 2005

[3] M. Martinis, N. Perković: Relativistic Modification of Newtonian Gravity Law, International Journal of New Technology and Research (IJNTR) ISSN:2454-4116, Volume-4, Issue-2, February 2018, pages 42-46 
[4] N. Perković,M.Stojić: Relativistic Equations of Motion Based on Equality of Heavy and Inertial Mass, International Journal of New Technology and Research (IJNTR) ISSN:2454-4116, Volume-4, Issue-11, November 2018, pages 44-47

[5] N.Perković, M. Stojić:Wawe Equation of a Particle with the Exponential Hamiltonian in the Gravitational and Electromagnetic Field, International Journal of New Technology and Research (IJNTR) ISSN:2454-4116, Volume-5, Issue-3, March 2019, pages 73-75 